\title{
Sternal Tuberculosis: A Case Report
}

\author{
Gyeong Hoe Kim, Sang Wha Kim \\ Department of Plastic and Reconstructive Surgery, Seoul National University Hospital, Seoul National University College of Medicine, Seoul, Korea
}

\begin{abstract}
The sternum is a very rarely involved site in extrapulmonary tuberculosis. Here, we present a case of sternal tuberculosis, and review the literature. An 84-year-old female patient visited Seoul National University Hospital presenting a $2 \times 3$ $\mathrm{cm}$ chest wound with sternum exposure. Chest magnetic resonance imaging revealed a sternal bone marrow signal corresponding to sternal osteomyelitis. Wound cultures yielded Mycobacterium tuberculosis, and tuberculosis polymerase chain reaction was also positive for $M$. tuberculosis. The wound was totally epithelized 1 month after debridement and initiation of oral anti-tuberculosis agents. Even though the prevalence of sternal tuberculosis is not high, when there is an unresolved infection or a chest wound that does not heal, sternal tuberculosis should be suspected and tests should be performed in order to obtain an early diagnosis and provide appropriate treatment.
\end{abstract}

Keywords: Wound healing; Tuberculosis; Osteomyelitis

\section{Introduction}

In about $15 \%$ of cases of tuberculosis occurring in immune-competent patients, the initial site is extrapulmonary. The sternum is very rare among extrapulmonary sites, accounting for less than $1 \%$ of all cases of tuberculous osteomyelitis, even in endemic countries, and falls under the differential diagnosis for chest wall masses [1-4].

The origin of sternal tuberculosis is not completely understood. It may be a late complication of pulmonary tuberculosis, or the result of reactivation of a latent focus of primary tuberculosis formed during hematogenous or lymphatic dissemination or of direct extension from mediastinal lymph nodes [5]. Here, we present a case of sternal tuberculosis, and review the literature.

\section{Case}

An 84-year-old female patient visited Seoul National University Hospital presenting a $2 \times 3 \mathrm{~cm}$ chest wound with sternum exposure (Fig. 1). Her past medical history included hypertension, diabetes, chronic kidney disease, and asthma, but no immunedeficient diseases. She had also previously received percutaneous coronary intervention for coronary artery occlusive disease.

She had undergone excision of a $3.8 \times 1.8 \times 0.7 \mathrm{~cm}$ sized irregular shaped yellowish soft tissue anterior chest mass with fluid collection at the department of thoracic surgery at another hospital about 5 months before. Gram stain and culture study results for the fluid had been negative, and biopsy of the mass had revealed acute and chronic suppurative inflammation. The wound became disrupted with pus discharge 2 months after excision, and the patient underwent debridement and primary closure by the hospital's plastic surgeons. The biopsy results of the secondary surgery presented a cystic lesion with chronic granulomatous inflammation and foreign body reac-

\footnotetext{
Case Report

Received: January 8, 2019

Revised: February 19, 2019

Accepted: February 19, 2019

Corresponding author:

Sang Wha Kim, M.D., Ph.D.

Department of Plastic and Reconstructive Surgery, Seoul National University Hospital, Seoul National University College of Medicine, 101 Daehak-ro, Jongno-gu, Seoul 03080, Korea

Tel: +82-2-2072-2375

Fax: +82-2-3675-7792

E-mail: sw1215@snu.ac.kr

This study was presented at The Wound Meeting 2018 on March 30, 2018, in Seoul, Korea.

This is an Open Access article distributed under the terms of the Creative Commons Attribution Non-Commercial License (http://creativecommons.org/licenses/by-nc/4.0/) which permits unrestricted non-commercial use, which permits unrestricted non-commercial use,
distribution, and reproduction in any medium, provided the original work is properly cited.

C) 2019 Korean Wound Management Society
} 
tion. Likewise, there were no specific findings in the Gram stain and culture studies. Three months later, the wound was

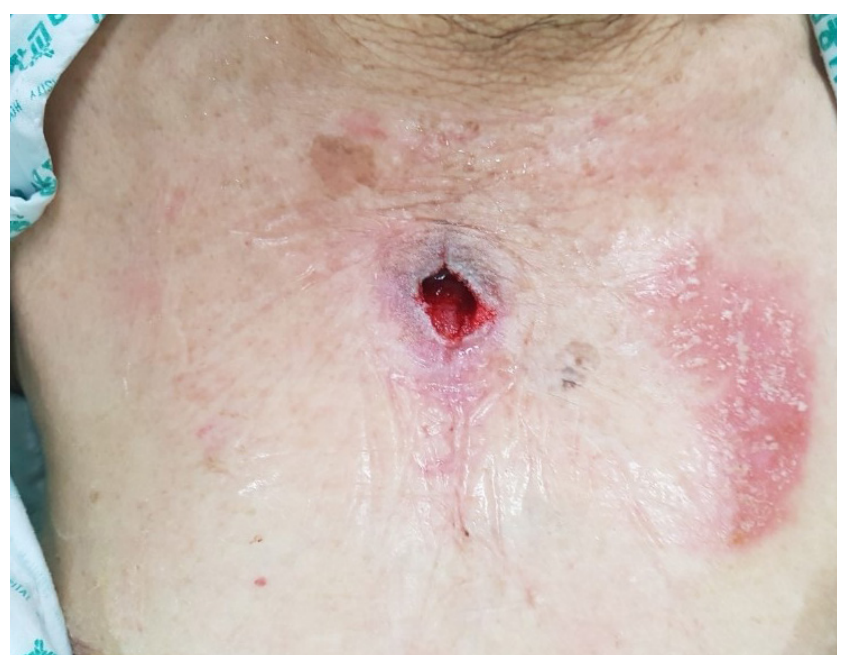

Fig. 1. Gross view of chest wound at admission. The wound had recurred twice after excision of an anterior chest mass. disrupted again with pus discharge, and the patient visited our hospital for further management.

At initial presentation to our institute, the wound did not have a severe odor and displayed only minimal discharge. The patient's white blood cell count was $10.04 \times 10^{3} / \mu \mathrm{L}$, erythrocyte sedimentation rate $59 \mathrm{~mm} / \mathrm{hr}$ and C-reactive protein $3.82 \mathrm{mg} / \mathrm{dL}$. There were no specific findings on simple chest radiographs. Chest computed tomography (CT) revealed no pulmonary or pleural lesions, but suspected mediastinal lymphadenopathy was visible (Fig. 2). Chest magnetic resonance imaging (MRI) revealed a sternal bone marrow signal corresponding to sternal osteomyelitis (Fig. 3).

Surgical debridement was performed, including curettage for sternal osteomyelitis to the extent where bone inflammation was visible, followed by negative pressure wound therapy. Biopsies of sternum, soft tissue, and internal mammary lymph nodes all revealed chronic granulomatous inflammation with non-caseating necrosis. Gram and acid-fast bacilli stains were
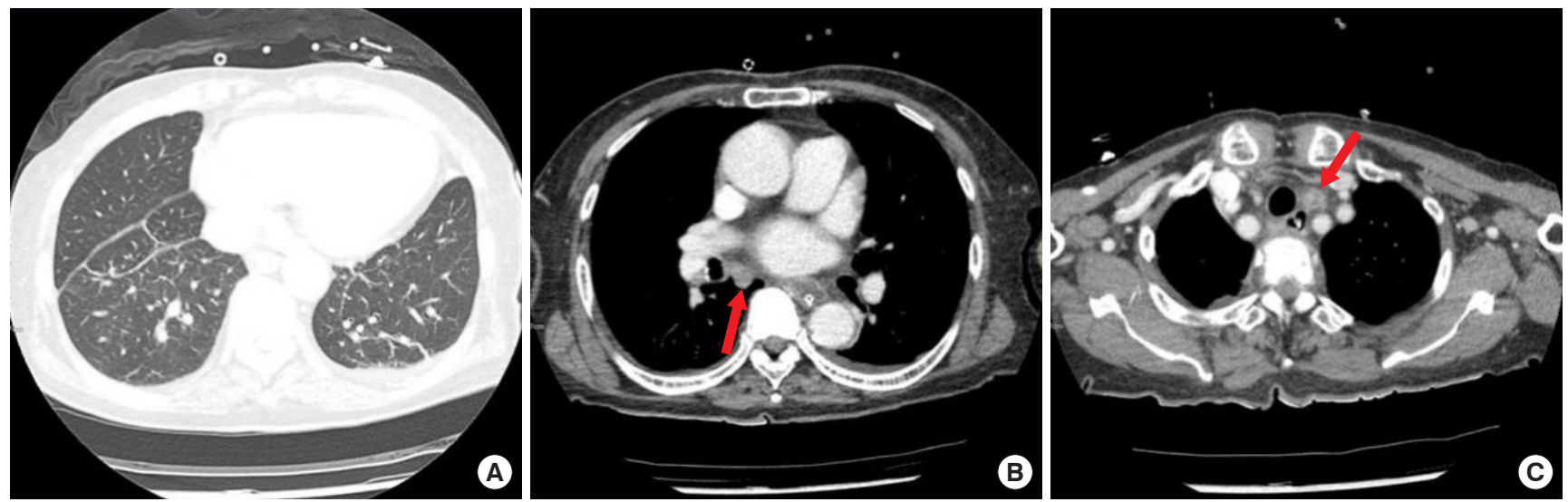

Fig. 2. Chest computed tomography (CT). (A) Chest CT showing no pulmonary or pleural lesions. (B, C) The arrow indicates mediastinal lymphadenopathy, probably due to primary sternal tuberculosis.
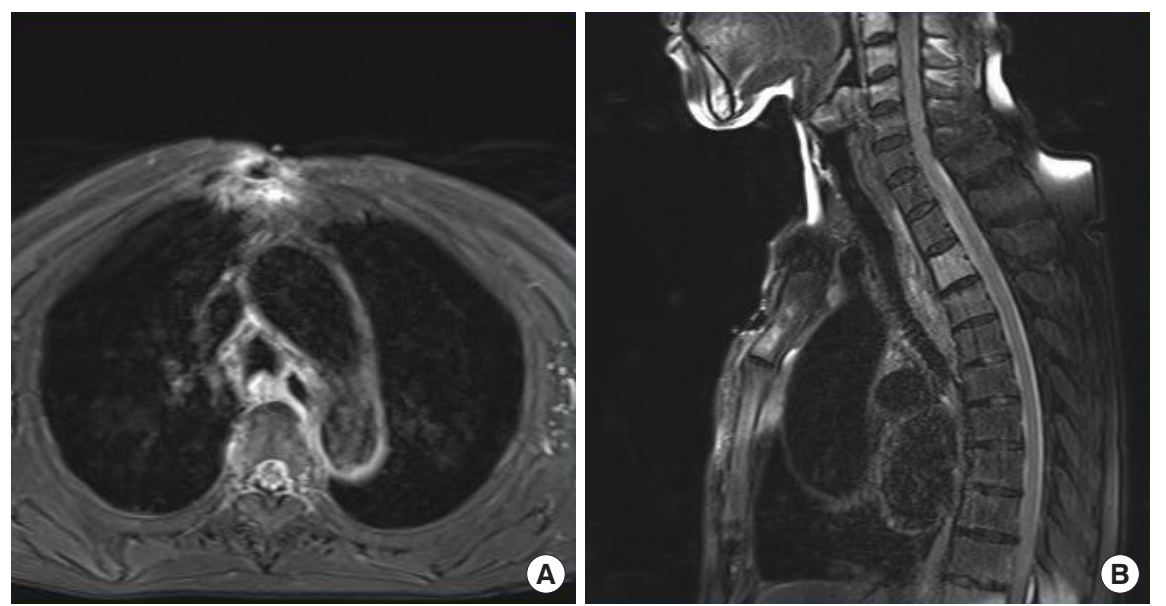

Fig. 3. Chest magnetic resonance imaging (MRI). Chest MRI showing sternal bone marrow signal corresponding to sternal osteomyelitis. (A) Axial view and (B) sagittal view. 
negative, but cultures yielded Mycobacterium tuberculosis. Tuberculosis polymerase chain reaction (PCR) was also positive for M. tuberculosis. Based on these results, oral administration of anti-tuberculosis agents (isoniazid, rifampicin, pyrazinamide, and ethambutol) was started. Immediate flap surgery for coverage of the defect at the time of debridement was considered but decided against, on the reasoning that if the cause of recurrent wound disruption was not precisely known, it would be likely to cause wound problems again after flap coverage. Also, general anesthesia was deemed risky due to the patient's old age and underlying diseases such as coronary artery occlusive disease, hypertension, diabetes, chronic kidney disease, and asthma.

After application of negative pressure wound therapy and administration of anti-tuberculosis agents, the therapeutic reaction was satisfactory, therefore negative pressure therapy was maintained without secondary surgery. Negative pressure wound dressings were regularly changed twice a week and maintained for about 3 weeks, after which granulation tissue had filled up and wound discharge had decreased enough to allow switching to simple dressings. White blood cell count and C-reactive protein levels decreased to $6.25 \times 10^{3} / \mu \mathrm{L}$ and $0.05 \mathrm{mg} / \mathrm{dL}$, respectively. The patient was discharged 1 week after removal of negative pressure wound therapy, and visited the outpatient clinic periodically for wound evaluation and dressing treatment. The wound was totally epithelized 1 month after initiation of oral anti-tuberculosis agents, but the patient was maintained on medication for 6 months.

\section{Discussion}

Sternal tuberculosis may cause non-specific clinical features of sternal swelling, pain and erythema, or constitutional symptoms such as fever, night sweat, and weight loss. As reported in several sporadic case reports including this one, it is important to suspect tuberculosis and carry out a diagnostic study if there is a non-resolving infection, or diagnosis can be delayed [5].

In sternal tuberculosis, the white blood cell count, erythrocyte sedimentation rate, C-reactive protein and lactate dehydrogenase levels are always elevated as in other infections. Thus, it is difficult to diagnose sternal tuberculosis with only laboratory blood tests, and radiologic tests such as CT and MRI may be helpful [1-3]. CT is the best modality for detecting osteolytic and sclerotic lesions, and MRI can detect early marrow and soft-tissue involvement. Histopathological and microbiological examinations are crucial for definitive diagnosis of sternal tuberculosis [2]. PCR can be helpful because results are obtained quickly and PCR findings are highly correlated with histological findings.

Whether or not surgical intervention is needed to treat sternal tuberculosis has yet to be established $[1,3]$. Some reported that the cure rate was approximately $95 \%$ with medical therapy only, while others reported that over $25 \%$ required concomitant surgical interventions [1]. Medication for sternal tuberculosis is a 6- to 9-month regimen according to the general treatment for extra-pulmonary tuberculosis [6,7]. The 6-month regimen is the same as the medication for pulmonary tuberculosis, but it can be extended to 9 months for sternal tuberculosis because it is difficult to determine the therapeutic response for bone infections [7]. The patients are given isoniazid, rifampicin, pyrazinamide and ethambutol for 2 months, followed by isoniazid and rifampicin for four to 7 months. In patients with sternal tuberculosis, isolation and identification of patient contacts are not required unless respiratory tuberculosis is present. Since clinical responses precede radiologic responses, therapeutic effectiveness can be evaluated by periodic clinical examination.

Without appropriate and effective management, sternal tuberculosis may lead to complications such as secondary infection, fistula formation, sternal fracture, and compression of large vessels and trachea [8]. M. tuberculosis infection can be relatively easily ruled out by microbiological tests, but if diagnosis is delayed patients can be exposed to unnecessary treatments, resulting in socioeconomic losses. In the present case the diagnosis of sternal tuberculosis was delayed, and the patient underwent repeated revision operations and tests.

Even though the prevalence of sternal tuberculosis is not high, when there is an unresolved infection or a chest wound that does not heal, sternal tuberculosis should be suspected and tests should be performed in order to obtain an early diagnosis and provide appropriate treatment.

\section{Conflict of interest}

No potential conflicts of interest relevant to this article are reported.

\section{Acknowledgments}

Gyeong Hoe Kim https://orcid.org/0000-0001-5992-2091

Sang Wha Kim https://orcid.org/0000-0003-0430-3458 


\section{References}

1. Calabro E, Pastorino U. Primary sternal tuberculosis mimicking a lytic bone tumor lesion. Monaldi Arch Chest Dis 2018; 88:931.

2. Hongsakul K, Chitrapazt N, Tubtawee T, et al. Primary sternal tuberculosis: a case report and literature review. Southeast Asian J Trop Med Public Health 2015;46:80-5.

3. Khan SA, Varshney MK, Hasan AS, et al. Tuberculosis of the sternum: a clinical study. J Bone Joint Surg Br 2007;89: 817-20.

4. Vasa M, Ohikhuare $C$, Brickner L. Primary sternal tuberculosis osteomyelitis: a case report and discussion. Can J Infect
Dis Med Microbiol 2009;20:e181-4.

5. Jain A, Chawla S, Kumar M. Sternal tuberculous osteomyelitis: a series of four cases. Int J Community Med Public Health 2017;2:288-92.

6. American Thoracic Society; CDC; Infectious Diseases Society of America. Treatment of tuberculosis. MMWR Recomm Rep 2003;52:1-77.

7. Tuberculosis Coalition for Technical Assistance. International standards for tuberculosis care (ISTC). 2nd ed. The Hague: Tuberculosis Coalition for Technical Assistance; 2009.

8. Sachdeva R, Sachdeva S, Arora S. Sternal tuberculosis. Ann Med Health Sci Res 2013;3(Suppl 1):S21-3. 